\title{
Local Excision of Rectal Cancer: Review of Literature
}

\author{
Piero Nastro Daniel Beral John Hartley John R.T. Monson \\ University of Hull Academic Surgical Unit, Castle Hill Hospital, Cottingham, UK
}

\section{Key Words}

Local excision, rectal cancer . Transanal excision . Endoscopic microsurgery, transanal $\cdot$ Rectal cancer

\begin{abstract}
In selected patients, local excision of rectal cancer may be an alternative to radical surgery such as abdominoperineal excision of the rectum or anterior resection. Local excision carries lower mortality and morbidity, without the functional disturbance or alteration in body image that can be associated with radical surgery. There are several techniques of local therapy for rectal cancer, with most experience being available in transanal excision. Transanal endoscopic microsurgery is also used but experience with this newer technique is limited. Patient selection is the most important factor in successful local excision, however specific criteria for selecting patients have not been universally accepted. Review of the published literature is difficult because of the variation in adjuvant therapy regimes and follow-up strategies, as well as results reported in terms of local recurrence and survival rates. There is increasing evidence to suggest that local excision should be restricted to patients with T1 stage rectal cancer without high-risk factors. The place for local excision in patients with T2 or high-risk T1 tumours requires prospective, randomised multi-centre trials comparing radical surgery with local excision, with or without adjuvant therapy.
\end{abstract}

Copyright $@ 2005$ S. Karger AG, Basel

\section{Introduction}

In the UK, colorectal cancer (CRC) is the third most common cancer after lung and prostate cancer in males, and is second only to breast cancer in females. In 1996 the UK incidence of CRC was 17,500 men and 16,800 women. In 1999, in England alone, there were 28,451 newly diagnosed cases. The lifetime risk of developing CRC is 5.7 and $4.9 \%$ for males and females, respectively, or in terms of odds, 1 in 18 men and 1 in 20 women. In 1998, there were 17,110 deaths due to CRC [1]. Here we review the literature relating to local excision of rectal cancer. Medline was searched using the terms 'local excision' and 'rectal cancer' to find relevant articles and the reference lists of these used to identify other key papers.

Modern therapy of rectal cancer is multidisciplinary but centres around surgery, which offers the only hope of cure. Radiotherapy and chemotherapy are used alone or in combination in an adjuvant, neo-adjuvant or palliative setting. Success of surgical treatment depends upon four variables: surgeon, operative technique, tumour biology and patient-related features. The principal goals in the management of any patient with rectal cancer are to provide the optimum chance for cure while maintaining quality of life and pelvic autonomic nerve function and avoiding a stoma [2]. The standard surgical therapy for rectal cancers has been radical resection, either with an abdominoperineal resection (APR) or an anterior resection (AR). Unfortunately resection of the rectum is a

Prof. John R.T. Monson

University of Hull Academic Surgical Unit

Castle Hill Hospital, Castle Road

Cottingham, HU16 5JQ (UK)

Tel. +44 1482 623225, Fax +44 1482 623274, E-Mail j.r.monson@hull.ac.uk 
Table 1. Variation in 5-year survival rates with TNM tumour stage

\begin{tabular}{ll}
\hline Stage & $\begin{array}{l}\text { 5-year survival } \\
\text { rate, } \%\end{array}$ \\
\hline T1/T2 - N0 & $80-90$ \\
T3/T4 - N0 & $60-70$ \\
T1/T2/T3/T4 - N1/N2 & $25-40$ \\
\hline
\end{tabular}

Table 2. Morbidity following anterior resection

\begin{tabular}{lc}
\hline Urinary dysfunction, \% & $7-68$ \\
Sexual impotence, \% & $15-100$ \\
Absent ejaculation, \% & $3-39$ \\
Dyspareunia, \% & $18-90$ \\
Anastomotic leak, \% & $5-10$ \\
Mortality, \% & 4 \\
\hline
\end{tabular}

major surgical procedure associated with significant morbidity (7-68\%), mortality (0-6.5\%), alteration in body image and sometimes distressing functional consequences for the patient [3]. Local excision of low rectal cancers has long been used as an alternative surgical option in patients thought to be unfit for major abdominal resection or who are unwilling to countenance a stoma [4]. Therefore local excision of distal rectal cancers has been explored as an option for selected patients with early stage rectal cancer [3].

The stage of disease at the time of diagnosis is the main prognostic factor in rectal cancer. The extent of invasion of the tumour through the bowel wall and nodal status governs the stage of disease and therefore the chances of survival. The node-bearing areas are not removed by local excision techniques rendering these procedures inadequate for node-positive tumours. However, even in nodenegative tumours, extension beyond the muscularis mucosa (T3) reduces the 5-year survival probability by approximately $20-30 \%$, making the T stage of critical importance in local excision [5] (table 1).

Many authors agree that local excision of properly selected rectal cancers is an acceptable alternative to radical surgery which may provide long-term survival with minimal morbidity, negligible mortality, and excellent functional results, as well as avoiding a colostomy [6-8]. The main issue is appropriate patient selection. However, there is little consensus about the specific criteria for local excision and the indications for adjuvant therapy in those patients. Review of the published literature would suggest that TNM stage T1 low-risk rectal cancer should be considered for transanal excision $[9,10]$. This article will review the controversy surrounding the optimal treatment of $\mathrm{T} 1$ high-risk and $\mathrm{T} 2$ rectal cancers.

\section{Historical Background}

At the turn of the century, perineal excision was the accepted approach for nearly all rectal cancers, but inconsistent surgical outcomes and high local recurrence rates promoted the search for alternative treatment. In 1908 Miles [11] described APR in the Lancet. Although not the first description, this report led to the establishment the operation in the UK and North America [12]. The acceptance of APR reduced recurrence and improved longterm survival but resulted in body image alteration due to the permanent stoma created. The introduction of circular staplers in the mid 1970s [13] and the concept of the low AR with total mesorectal excision, as championed by Heald et al. [14] in the early 1980s, allowed survival and tumour recurrence rates comparable to APR without the need for permanent stoma formation [15]. Unfortunately, low AR is not without significant morbidity, with sexual and urinary dysfunction being common (table 2). Operative mortality rates following the two approaches are broadly similar at around 4-6\% [4].

In recent years there have been remarkable advances in the treatment of rectal cancer, stimulated by a greater understanding of tumour biology and aided by the development of diagnostic tools that allow the surgeon to select the most suitable tumours for the various treatment strategies available. The role of local excision has evolved during the latter part of the 20th century. As abdominal surgery became safer, local excision was usually considered only in the palliative setting or for patients considered a high anaesthetic risk. More recently, as preoperative tumour-staging methods have improved, local excision has been performed with curative intent in selected patients [6].

\section{Tumour Staging}

Rectal cancer is staged preoperatively using the Dukes' or TNM classifications in order to predict the likelihood of a curative resection, based upon the depth of rectal wall 
penetration and the presence of lymphatic and distant metastases. The presence of lymph node metastasis, confirmed postoperatively by histopathological examination, mandates radical surgery if the intention is for a curative resection and will usually lead to the administration of adjuvant chemotherapy. Methods of staging comprise the following.

\section{Digital Rectal Examination}

Digital rectal examination is the simplest method of preoperative staging, but is highly surgeon-dependent. Nicholls et al. [16] reported the accuracy rate amongst colorectal specialists and trainees as $44-83 \%$ for the assessment of the depth of invasion ( $\mathrm{T}$ stage), and 57$67 \%$ for the assessment of lymph node involvement $(\mathrm{N}$ stage; table 3).

\section{Endorectal Ultrasound}

Endorectal ultrasonography (ERUS) is the most useful preoperative method to stage the depth of invasion of rectal lesions; in fact the T stage can be measured accurately in $82-93 \%$ (fig. 1), although over-staging has been reported. The assessment of lymph node involvement by ERUS is less reliable with difficulty in distinguishing between malignant deposits and reactive changes. Accuracy for this varies between 65 and $81 \%$ [4] (table 3). Previous biopsies or irradiation may reduce the accuracy of assessment and a stenosing lesion may limit access of the ultrasound probe. Newer three-dimensional imaging technology may improve the accuracy of ERUS staging and overcome some of the difficulties associated with stenotic lesions [4].

\section{Computed Tomography}

Computed tomography (CT) scanning for rectal cancer, which lies within the bony pelvis, is not as accurate as ERUS for the assessment of either local invasion (66$88 \%$ accuracy) or nodal spread (60\% accuracy; table 3$)$. The main value of CT imaging is in the excellent definition of regional and distant spread [4].

\section{Magnetic Resonance Imaging}

Magnetic resonance imaging (MRI) has accuracy rates comparable to CT scanning (table 3) and in some centres MRI is used in preference to CT for rectal cancer. Some evidence suggests MRI is superior to both CT and ERUS [17]. Endorectal MRI coils may improve accuracy but their use depends on operator expertise which is not yet widely available [4]. Also, the examination takes longer than ERUS and the relatively large endorectal coil not

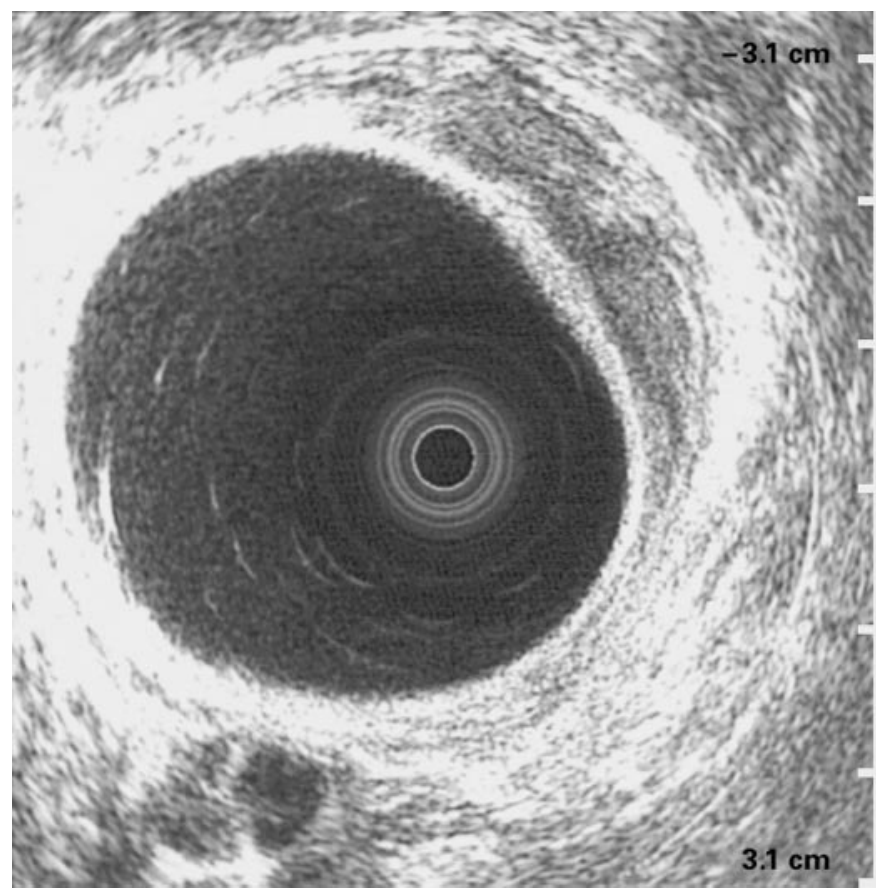

Fig. 1. Endorectal ultrasound image demonstrating a $\mathrm{T} 2$ rectal tumour in the upper-right quadrant.

Table 3. Diagnostic accuracy of pre-operative staging modalities

\begin{tabular}{lll}
\hline Staging modality & T Stage & N Stage \\
\hline Digital rectal examination, \% & $44-83$ & $57-67$ \\
Endorectal ultrasound, \% & $82-93$ & $65-81$ \\
Computed tomography, \% & $66-88$ & 60 \\
Magnetic resonance imaging, \% & $68-90$ & 62 \\
\hline
\end{tabular}

infrequently results in failure of examination due to patient discomfort or a bulky, stenosing tumour $[18,19]$. Endorectal coil MRI and ERUS have shown similar results, so the use of endorectal coils would seem only to be justified in patients in whom ERUS has yielded doubtful results [17].

\section{Case Selection}

The need for appropriate patient selection is the main issue in local excision of rectal cancers. The high local recurrence rates and compromised survival reported in some series has convinced many surgeons to restrict the use of local excision in otherwise healthy patients to only 
Table 4. Risk of nodal involvement by TNM tumour stage

\begin{tabular}{lllll}
\hline & T1 & T2 & T3 & T4 \\
\hline Nodal involvement, \% & $0-12$ & $12-28$ & $36-66$ & $53-79$
\end{tabular}

Table 5. Local recurrence following local excision of rectal cancer

\begin{tabular}{lc}
\hline T Stage & $\begin{array}{l}\text { Local recurrence, } \% \\
\text { (range) }\end{array}$ \\
\hline Overall & 13.7 \\
T1 & $9.7(0-24)$ \\
T2 & $25(0-50)$ \\
T3 & $38(0-100)$ \\
\hline
\end{tabular}

the most favourable rectal cancers [6]. Several retrospective studies have demonstrated that tumour control in properly selected patients treated with local excision is comparable to that which is anticipated after radical surgery. However, the case mix within these series is variable and results after local excision differ greatly between centres [20].

\section{Pre-Operative Staging}

Local excision does not remove the draining lymph nodes, so the surgeon needs to be confident of the absence of nodal spread before undertaking such a procedure. The staging modalities described above have limits to their accuracy which are greater in regard to the assessment of lymph node involvement. However, the depth of wall invasion has been shown to be related to lymph node metastasis [3, 21, 22] (table 4), a higher risk of local recurrence following local excision [4] (table 5), as well as survival (table 1). As such, wall invasion as classified by the TNM T stage during pre-operative assessment provides a good estimate of the probability of nodal involvement and thus the feasibility of local excision. An additional pathologic classification sub-classifies $\mathrm{T} 1$ tumours into $\mathrm{Sm} 1, \mathrm{Sm} 2$ or $\mathrm{Sm} 3$ depending upon the level of invasion of the submucosa and has also been shown to correlate with lymph node metastasis and local recurrence [23]. Overstaging of the tumour may lead to suitable patients not being offered local excision. The major concern, however, is the risk of understaging of the tumour by the currently available imaging modalities leading to inappropriate selection for local excision.
There is general consensus that the use of local therapy with curative intent should not be considered for tumours that are advanced (T3 or T4) due to the high risk of lymph node involvement. However, clear suggestion of nodal involvement on the pre-operative imaging, whatever the $\mathrm{T}$ stage, will also necessitate radical surgery.

\section{Clinico-Pathologic Variables}

These other variables contribute towards the assessment of the suitability of the tumour for local excision by determining whether the patient is at higher risk of relapse than another patient with a tumour of the same stage. For example, the histologic grade of the tumour, based upon the degree of cytologic differentiation of the tumour cells, reflects its aggressiveness. The probability of lymph node metastases for a T1-G1 well-differentiated tumour is $0 \%$, with recurrence rates of only $0-3 \%$; compared with $12 \%$ nodal metastases and $10-63 \%$ recurrence for a T1-G3 poorly differentiated tumour [4].

Lymphovascular invasion is believed to be a pre-metastatic event carrying a greater risk of nodal spread and increasing local recurrence by more than 25\% [4]. Mucinous and ulcerated tumours are often excluded from local excision because they are believed to behave in a more aggressive manner [4].

Tumour size may also be of relevance with several studies suggesting that recurrence is higher following local

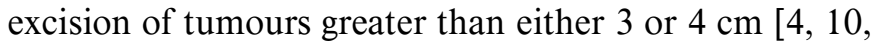
$22,24]$. Tumours that occupy over $40 \%$ of the circumference of the rectal wall may also be excluded from local excision based upon the possibility of technical difficulties in closure of the defect (although it may not always be necessary to close the defect).

Age has not been shown to be an important criterion for patient selection [3]. However, we clearly balance the risk of local recurrence against a patient's anticipated lifespan, based upon their co-morbidities. Thus we would wish to be more aggressive in younger, fitter patients, judging each case on its individual merits. Other factors such as chromosomal ploidy and genetic markers (e.g. p53, bcl2, c-myc, k-ras) may influence prognosis within each pathologic stage and are being actively investigated. If shown to be significant with respect to prognosis, recurrence or the likelihood of nodal metastasis they will prove particularly useful in selecting low-risk rectal cancer patients suitable for local excision.

Currently therefore, commonly utilised criteria for local excision of rectal cancer are: TNM stage T1-T2, N0, $\mathrm{M} 0$, histological grading $\mathrm{G} 1$ or G2 and tumours up to 3$4 \mathrm{~cm}$ in size which are technically accessible [25]. 


\section{Methods}

There are several techniques for local therapy of rectal cancer which include: transanal excision, dorsal approaches (York-Mason or Kraske procedures), transanal endoscopic microsurgery (TEM), endocavity radiation and transanal fulguration [8]. For most groups, conventional transanal excision is favoured for its low rate of complications, promising outcomes, and the ability to secure tissue for pathological examination [6]. In recent years TEM, following good results in the excision of rectal adenomas, has become more widely accepted as a technique for local excision of rectal cancer, however experience is still limited [4].

\section{Transanal Excision}

For transanal excision the patient is best positioned prone for anterior wall tumours or in the lithotomy position if the tumour is posterior. Bowel preparation and preoperative antibiotics are given in all patients. Anaesthesia may be general or regional (e.g. pudendal nerve block). Retractors, preferably with a fibre-optic light source, are used to visualize the lesion and adrenaline may be injected around the lesion to prevent bleeding. Traction sutures are conventionally placed around the lesion and the line of excision marked using diathermy, aiming for a margin of at least $1-2 \mathrm{~cm}$. Full-thickness excision is performed taking care to protect the vagina or prostate. After haemostasis, the defect may be closed with transverse absorbable sutures or, if below the peritoneal reflection, left open with the perirectal fat exposed. Fibrin gauze may be positioned in the rectum, depending on the surgeon's preference, following checking by proctoscopy. Postoperative analgesia requirements are minimal and antibiotics may be continued for $24 \mathrm{~h}$. Normal diet may be taken by the next day and in-patient stay is often as little as 2 days. The complication rate is very low $(0-22 \%)$ with the most commonly occurring being: bleeding, local sepsis, urinary infection or retention, faecal incontinence and rectovaginal fistula [4]. Transanal excision is not usually performed for lesions beyond $10 \mathrm{~cm}$ from the dentate line due to technical difficulties such as access to the lesion with standard instrumentation.

\section{Transanal Endoscopic Microsurgery}

TEM is a relatively new technique first described in 1985 [26]. It is performed through a 40-mm operating rectoscope with carbon dioxide insufflation to obtain vision. Operating instruments are introduced through sleeves in the same manner as laparoscopic surgery and the tumour is excised using bipolar diathermy. The preparation and positioning of the patient is the same as for transanal excision. Disadvantages of TEM include an initial capital outlay for equipment in the region of GBP 50,000, a long learning curve similar to that of laparoscopic surgery and difficulty in haemostasis of large vessels. Although, in theory, lesions up to $25 \mathrm{~cm}$ from the anal verge may be excised by TEM, it is usually reserved for tumours in the extra-peritoneal rectum due to the risk of perforation and formation of pneumoperitoneum, as well as technical difficulties. This limits the technique to lesions up to $20 \mathrm{~cm}$ on the posterior wall, $15 \mathrm{~cm}$ laterally and $12 \mathrm{~cm}$ on the anterior wall. Also, the most distal lesions are often unsuitable for TEM excision as it is difficult to maintain an adequate seal around the rectoscope and insufflation pressure is lost. To date, experience with TEM excision of rectal cancers is limited. However, TEM gives a magnified view of the operative field which enables precise excision of the tumour whilst maintaining a constant view of the margin, which may facilitate a more accurate and oncologically sound excision. Complications are infrequent but include bleeding, intraperitoneal perforation, fistula formation, incontinence and wound breakdown.

\section{Posterior Approaches}

The posterior approaches for local excision of rectal cancers via a trans-sacral (Kraske) or trans-sphincteric (York-Mason) approach have been largely abandoned. These techniques had an advantage over conventional transanal excision in that the perirectal nodes could be removed for histopathological examination. However, they are associated with a significant rate of faecal fistula formation, wound breakdown and incontinence [4, 27].

\section{Palliative Procedures}

Other methods for local control include electrocoagulation, endocavitary irradiation, photodynamic therapy, cryosurgery and laser ablation, but they have been abandoned in the potentially curative setting as they are unable to obtain a pathological specimen for staging and give poor results in terms of survival. These are ablative procedures largely limited to palliation in modern colorectal surgical practice.

\section{Results}

The results of local excision of rectal cancer vary widely between centres. Most of the data derive from singleinstitution retrospective case series and the different tech- 
niques of local excision have never been subjected to analysis in a randomised controlled trial. Most reports correctly highlight rates of local recurrence as the key comparator but will often also discuss survival. Survival in itself is probably not useful as an outcome measure for local excision in early stage rectal cancer as it may to a large extent be dependent upon the rate of local recurrence.

\section{Local Excision Alone}

A review conducted by Sengupta and Tjandra [4] published in 2001 reported local recurrence rates following local excision ranging from 0 to $32 \%$. The wide variation is easily explained by differences in patient selection, surgical technique, duration and frequency of follow-up. In addition the histopathological criteria used to assess the adequacy of surgical excision vary between institutions, as do the policies for re-operation following an inadequate resection (i.e. repeated local excision vs. radical resection). The review included 958 patients in 22 studies and the stage-related local recurrence rates are shown in table 5 [4].

The favourable results reported by Blair and Ellenhorn [10] (not included in the review by Sengupta and Tjandra [4]) demonstrate the importance of patient selection. In 20 patients with Tis or T1 lesions and favourable histology, they achieved $0 \%$ recurrence and $0 \%$ cancer-related mortality at 60 months follow-up following transanal excision.

In contrast, Garcia-Aguilar et al. [20] (also not included in the review) report on 82 patients with T1 or T2 tumours following transanal excision. After 54 months follow-up they report $18 \%$ recurrence in $\mathrm{T} 1$ tumours and $37 \%$ in $\mathrm{T} 2$ tumours. The same researchers also compared their results following local excision to those after radical surgery in a retrospective review, which is the only large study of its kind. Patients with tumours that were pathologically identified as $\mathrm{T} 1$ or $\mathrm{T} 2$ and who did not receive adjuvant chemoradiation were selected from a database covering a 10-year period. Those with positive resection margins, tumours removed by endoscopic polypectomy or those with incomplete data or follow-up were excluded. There remained 108 local excisions $(\mathrm{T} 1=69, \mathrm{~T} 2=39)$ with 4.4 years follow-up and 153 radical resections $(\mathrm{T} 1=$ $30, \mathrm{~T} 2=123$ ) with 4.8 years follow-up. There were significant differences between the 2 groups in the distance of the lesions from the anal verge (average $3 \mathrm{~cm}$ lower in local excision patients, as might be expected as the highest lesions would not be accessible for local excision), the size of the tumours (smaller in local excision) as well as the stage bias shown above. There were 19 patients excluded on the basis of positive resection margins following local excision but no patient had a positive margin following radical resection. Bearing in mind these inequalities, the results show a high rate of local recurrence after local excision $(\mathrm{T} 1=18 \%, \mathrm{~T} 2=47 \%)$ compared to radical surgery $(\mathrm{T} 1=0 \%, \mathrm{~T} 2=6 \%)$. There was also a higher rate of overall recurrence, including distant metastases, in the local excision group $(\mathrm{T} 1=21 \%, \mathrm{~T} 2=47 \%)$ compared to radical surgery $(\mathrm{T} 1=9 \%, \mathrm{~T} 2=16 \%)$, which was statistically significant for T2 lesions. The 5-year cancer-specific mortality was similar between the 2 groups but there was a significantly lower 5-year overall survival rate in the local excision group for T2 lesions ( 65 vs. 81\%, p = 0.03) [3].

This study would seem to point towards two key conclusions: firstly, when judged on overall survival, patients with T2 tumours are disadvantaged by local excision alone. Secondly, local and overall recurrence is significantly higher following local excision in $\mathrm{T} 2$ tumours. There is a clear implication that $\mathrm{T} 2$ lesions in particular are not suitable for local resection. Histopathological characteristics such as differentiation, lymphovascular invasion and mucinous tumours do not form part of the selection criteria in this study, only positive resection margins, which are not available to the surgeon pre-operatively. The authors state that the rate of local recurrence remained comparatively high even after the exclusion of patients with unfavourable histology but the methodology and data for this are not included. This and the other limits to this study in terms of the control group chosen, as discussed above, make it difficult to draw definitive conclusions. As the authors acknowledge, debate in this area is likely to continue until there is a prospective, randomised clinical trial comparing radical surgery to local excision [3].

\section{Local Excision with Adjuvant Therapy}

The use of radiotherapy and chemotherapy represents an additional variable in any assessment of local excision for rectal carcinoma. The indications for neoadjuvant (pre-operative) or adjuvant therapy and local excision vary between centres but such treatments are increasingly being offered for $\mathrm{T} 2$ tumours and $\mathrm{T} 1$ tumours with poor histopathologic characteristics, due to the poor results achieved by local excision alone. Adjuvant or neoadjuvant chemoradiotherapy regimes do appear to improve results following local excision when compared to historical series of surgery alone, but there is a lack of good, controlled data. Variations in the results from single institution studies that include these therapies are partly due to differences in the timing and dose/fractionation of the 
therapy utilized. Overall, these uncontrolled studies indicate that adjuvant chemotherapy reduces local recurrence and improves survival in high-risk tumours [28-39].

The Cancer and Leukaemia Group B researchers report a multi-institutional phase-2 trial with local excision alone for T1 tumours and local excision followed by chemoradiotherapy for T2 tumours. The median follow-up period was 4 (range 1.8-7.7) years. Fifty-nine patients with T1 lesions underwent local excision alone and their estimated 6-year overall survival rate was $87 \%$ with disease-free survival of $83 \%$. Fifty-one patients with T2 tumours were treated with local excision followed by chemoradiotherapy with 85\% 6-year overall survival and $71 \%$ disease-free survival. Disease-free survival is continuing to decline after 5 years and long-term follow-up is awaited [40]. This may reflect a change in the pattern of local recurrence in rectal cancer induced by radiotherapy, i.e. late local recurrence, which has been documented previously [41].

The Radiation Therapy Oncology Group report a phase-2 trial of local excision in 65 patients selected on the basis of T1-3 lesions with no evidence of nodal metastases on CT imaging; less than $4 \mathrm{~cm}$ in diameter and occupying less than $40 \%$ of the rectal wall circumference; who were judged to have required APR if a local excision was not performed. Those patients identified with highrisk lesions following local excision (T2, T3, high grade, lymphovascular invasion, size $>3 \mathrm{~cm}$ or elevated carcinoembryonic antigen) were treated with adjuvant chemoradiotherapy (with higher dose radiotherapy in the case of doubtful margins of $<3 \mathrm{~mm}$ ). Local recurrence rates (local recurrence alone or in combination with distant metastases) were $4 \%$ for T1, 16\% for T 2 and $23 \%$ for T 3 cancers at a minimum of 5 and a median 6.1 years follow-up. The data for local recurrence alone were 4,8 and $0 \%$, respectively, and for overall recurrence (local or distant) 7.4, 20 and $31 \%$. Overall mortality was $25 \%$ with no statistically significant differences in either overall survival or disease-free survival between the treatment groups [42].

There have also been reports of the use of neo-adjuvant therapy to downstage tumours before local excision. The earliest study, in 1989, used pre-operative radiotherapy (35 Gy over 3 weeks) in 25 patients, over half of whom were considered unfit for radical surgery. The study protocol included postoperative interstitial radiotherapy (20 Gy for T1 and 25 Gy for T2-3 lesions) and pre-operative staging was based upon examination under anaesthesia rather than radiologic imaging. However, $92 \%$ of patients showed a reduction in tumour diameter and 2 patients showed a complete response with a pathologic stage of pT0. Local recurrence rates were $0 \%$ for T1, $25 \%$ for T2 and 30\% for T3 lesions [43].

Mohiuddin et al. [44] report on 48 patients with T1-3 lesions, 15 of whom had large T3 cancers and underwent local excision due to contraindications to radical surgery. In this study staging included ERUS and in some cases endorectal-coil MRI. The total radiation dose was 40$45 \mathrm{~Gy}$ with a boost given in fixed tumours. After a median follow-up of 40 months the local recurrence rates were 11 , 0 and $67 \%$ for T0-1, T2 and T3 lesions, respectively. The 5 -year survival rate was $90 \%$ for T0-1, 89\% for T2 and $50 \%$ for T3 lesions [44]. A second report from the same department specifically analysed patients with tumours in the distal $2 \mathrm{~cm}$ of the rectum, with the intention of establishing that pre-operative high-dose radiotherapy enhances the options for sphincter-preserving surgery. All patients received pre-operative radiotherapy as in the first study and were then re-staged clinically and, as appropriate, with ERUS or MRI. No mention is made of any criteria used to decide between the several different surgical approaches, both radical and local, that are included in the study. It is stated that local excision was initially only considered in patients with cardiorespiratory problems who were unsuitable for radical resection, but the criteria were subsequently expanded to healthy patients with tumours of $<1.5 \mathrm{~cm}$, confined to the rectal wall. Twentytwo of 70 patients had full-thickness local excision. Two of these $(9 \%)$ developed local recurrence after a median follow-up of 4 years and actuarial 5-year survival was 95\% [45]. It is difficult to interpret these results with any degree of certainty as they are not sufficiently separated from the results of other surgical techniques and there is likely to have been bias in the selection of patients for local excision.

Kim et al. [46] report a series of patients who received neo-adjuvant chemoradiotherapy before local excision. This is a retrospective review which included 26 patients with T2-3 lesions, all but 1 of whom were staged with ERUS. They received 45 Gy in 25 fractions with continuous infusion 5FU 5 days/week, followed by re-assessment for clinical response with sigmoidoscopy and digital rectal examination. Twelve patients also had a further ERUS examination but the authors comment that the accuracy of $\mathrm{T}$ staging after chemoradiotherapy by this technique was only $\sim 30 \%$. Only lesions that had complete clinical response (defined as no evidence of residual disease) were initially considered for local excision, although patients with partial response (decrease in size of at least 50\%) who refused or were unfit for radical surgery were also included. Pre-therapy staging was: T2N0 = 5, 
$\mathrm{T} 3 \mathrm{~N} 0=13$ and $\mathrm{T} 3 \mathrm{~N} 1=7$. Four were deemed partial and 22 complete responders on clinical evaluation. Following histopathological examination the number of complete responders (i.e. no residual tumour; with pathological partial response defined as evidence of residual tumour) reduced to 17 and 1 patient had a positive margin. Median follow-up was 19 months and 1 patient had both local (intramural) and regional (inguinal lymph node) recurrence. This patient was a partial responder who initially refused the radical surgery recommended to all partial responders, but later underwent salvage abdominoperineal resection and inguinal lymph node dissection. The rate of complete response to chemoradiotherapy in this study is notably high at $65 \%$. In their discussion the authors state that complete pathologic responses can be expected in up to $31 \%$ of cases, but do not comment on their own higher rate [46].

Clearly, the data are limited with respect to neo-adjuvant therapy prior to local excision but they do suggest that it may have a role to play. For both neo-adjuvant and adjuvant therapy, the data are usually retrospective, single institutional experience in which there are few defined selection criteria and limited standardisation of surgical technique with variation in chemotherapeutic regimes.

\section{Transanal Endoscopic Microsurgery}

Results for this new technique are usually reported separately from conventional methods of local excision and frequently include results for benign rectal lesions. A full review of this emerging technology lies beyond the scope of this article. It is generally accepted that TEM preserves anorectal function with limited morbidity and mortality, but results in terms of recurrence and survival results vary too greatly between centres for it to be fully accepted at this time. Again, differences in patient selection, adjuvant therapy and follow-up policies will account for some of the variation. As the technique is simply an alternative method of providing full-thickness rectal excision it would seem unlikely, all other things being equal, that results will prove to be significantly different to conventional local excision techniques. Indeed, the binocular stereoscope gives the surgeon a three-dimensional view with depth of perception which facilitates precise excision of the tumour and maintains a view of the margin and may prove to be an oncologically superior technique.

Sengupta and Tjandra [4] included in their review 11 TEM series from 1992 to 1998 including 314 patients and found local recurrence rates varying from 4.2 to $25 \%$ and overall survival rates from 62 to $100 \%$ at an average follow-up of 35-60 months. As alluded to above, these stud- ies included some T3 patients, variably administered adjuvant radiotherapy and had variable follow-up duration, making overall evaluation of the technique difficult [4].

Analysis of these initial results leads many authors to conclude that TEM has a useful place in the management of adenomas of the rectum, in particular if they are sessile and are located in the mid and upper thirds of the rectum, as well as for carefully selected patients with carcinoma. It has low morbidity and mortality, a short hospitalization period and the potential for greater precision of resection than other techniques. However, the results of larger series of rectal cancer treated by TEM is required, with appropriate patient selection and complete follow-up, before the technique will be fully accepted. Even then, the cost of the equipment and the specialised surgical expertise required may limit the procedure to tertiary referral centres.

\section{Recurrence}

The ability to successfully perform salvage surgery in patients with local recurrence following local excision is important. It is thought that these recurrences may be biologically different to recurrences after radical surgery. This may be due to the greater proportion of recurrences occurring within the rectal wall, rather than extra-luminal pelvic recurrence, presumably related to mesorectal nodal disease not addressed by the local resection. For example Mellgren et al. [3] report 17 (68\%) rectal wall recurrences and $8(32 \%)$ exclusively extra-rectal recurrences. However, the number of attempted salvage procedures following local excision reported in the literature is low and disease-free survival ranges from 20 to $100 \%$ with variable follow-up duration [4]. A further variable to take into account is the salvage procedure undertaken. Some of the rectal wall recurrences have been dealt with by repeat local excision instead of proceeding directly to radical surgery. Patient-related factors as well as the type of recurrence will play a role in this decision. A recent report by Friel et al. [47] included 29 patients who underwent salvage surgery (APR, low AR or pelvic exenteration) following local excision of T1 or T2 rectal cancer. Of these, $90 \%$ involved the rectal wall with only $10 \%$ being purely extra-rectal. They found the stage of the recurrence to be more advanced than the original primary in $93 \%$ despite the use of preoperative radiotherapy in $12(44 \%)$ of patients. The disease-free survival was $59 \%$ following salvage surgery at a mean follow-up of 39 months, however 6 
( $21 \%)$ of the operations were non-curative due to positive margins or unresectable liver metastases. Eleven patients developed further recurrences after salvage surgery, only 2 of which occurred in the non-curative group. Interestingly, they found that the disease-free survival following salvage surgery for those with primary tumours that had favourable histology was higher than for those with unfavourable histology of the locally excised primary (68 vs. 29\%) [47]. These results indicate that delayed radical surgery for recurrence after local excision may not provide the same chance of cure as a radical resection performed as the initial treatment and emphasises the importance of careful patient selection. Whether locally excised tumours found to display high-risk characteristics should have immediate radical surgery is a question that has not yet been answered. Early detection of recurrence is essential to allow surgical salvage. As extra-rectal recurrences occur in a significant proportion of patients ERUS or MRI must be a key component of follow-up following local excision.

\section{Conclusions and Current Recommendations}

The published literature leaves several unanswered questions regarding local excision for rectal cancer. The ongoing concerns regarding the morbidity, mortality and quality of life issues associated with radical resection has led the surgical community to revisit local therapy as a means of managing selected patients with rectal cancer. It is clear that accurate staging of rectal cancer will, in the long term, be the key to the success of local excision as it will allow appropriate selection of patients. ERUS is currently the most effective method of assessing the depth of wall invasion by the tumour but it is less reliable at predicting lymph node involvement, which is a crucial factor. $\mathrm{CT}$ and MRI have similar limitations. Improved imaging with 3D-ERUS or endorectal coil MRI may improve accuracy but neither technology is at present widely available. A particular limitation is staging following neo-adjuvant chemoradiotherapy. This applies to clinical examination and imaging modalities which can be misread and also to histopathological staging, for which there is not yet a consensus upon how to classify unique post-chemoradiotherapy features or upon the prognostic value of posttreatment staging.

Appropriate patient selection is the critical requirement for local excision of rectal cancer. Currently, with less than perfect pre-operative prediction of nodal involvement, tumour stage is the most useful discriminatory factor. Only T1 or T2 lesions without evidence of nodal or distant spread should be considered for local excision in otherwise fit patients. In addition, large tumours $(>3-$ $4 \mathrm{~cm}$ ) occupying $>40 \%$ of the rectal circumference, or with poor prognostic factors such as mucinous tumours, high histological grade, lymphovascular invasion or ulceration should also be excluded from local excision alone due to higher than acceptable recurrence rates. We await, as in radical surgery, the emergence of other biological factors which are predictive of stage, recurrence and survival.

Current data suggest that $\mathrm{T} 1$ tumours with no high-risk factors may be suitable for local excision alone with good results, although data comparing local excision to radical surgery in a randomised, controlled fashion do not exist. T2 tumours or T1 tumours with high-risk factors do less well following local excision alone and may benefit from adjuvant therapy if local excision is undertaken. The data supporting this approach are limited and suffer from variability in patient selection and treatment regimes. There are two phase- 2 trials but long-term data for these are not yet available. The data supporting neo-adjuvant therapy in local excision are even more limited and require further evaluation.

The best method of local excision is also open to question. Transanal excision produces good results with minimal complications. It is possible that TEM excision will allow a more oncologically sound full-thickness excision with equally minimal complications but this has yet to be proven. Whatever surgical approach is chosen, given that the risk of local recurrence following local excision is significant, post-operative follow-up should be at least as intense as that following radical surgery to ensure early relapse detection. It should include radiologic imaging of the pelvis and liver as well as endoscopic examination of the rectum and possibly endorectal imaging. It should also be extended to cover the risk of late recurrence if the patient has received radiotherapy.

In summary, there is a clear need for well-designed, randomised controlled trials to answer the questions surrounding local excision of rectal cancer. The available evidence suggests that low-risk T1 tumours may be treated by local excision alone and $\mathrm{T} 2$ or high-risk $\mathrm{T} 1$ tumours will benefit from adjuvant chemoradiotherapy following local excision. However, many would still consider it best practice to perform radical surgery for $\mathrm{T} 2$ tumours and high-risk T1 tumours if the patient is a suitable candidate. 


\section{References}

1 National Statistics Online: Cancer Trends. National Statistics website: www.statistics.gov.uk 2003. Ref Type: Electronic Citation.

-2 Spencer MP: Transanal excision for T1 and T2 rectal cancer - Efficacy of local resection vs. adjuvant therapy. Extended abstract. Swiss Surg 2001;7:275-277.

$\checkmark 3$ Mellgren A, Sirivongs P, Rothenberger DA, Madoff RD, Garcia-Aguilar J: Is local excision adequate therapy for early rectal cancer? Dis Colon Rectum 2000;43:1064-1071.

$\checkmark 4$ Sengupta S, Tjandra JJ: Local excision of rectal cancer: what is the evidence? Dis Colon Rectum 2001;44:1345-1361.

5 Wexner SD, Rotholtz NA: Surgeon influenced variables in resectional rectal cancer surgery. Dis Colon Rectum 2000;43:1606-1627.

-6 Rothenberger DA, Garcia-Aguilar J: Role of local excision in the treatment of rectal cancer. Semin Surg Oncol 2000;19:367-375

7 Balani A, Turoldo A, Braini A, Scaramucci M Role of curative local excision in rectal cancer (in Italian). Ann Ital Chir 1999;70:713-720.

$>8$ Visser BC, Varma MG, Welton ML: Local therapy for rectal cancer. Surg Oncol 2001;10: 61-69.

-9 Ambacher T, Kasperk R, Schumpelick V: Effect of transanal excision on rate of recurrence of stage I rectal carcinoma in comparison with radical resection methods (in German). Chirurg 1999;70:1469-1474.

10 Blair S, Ellenhorn JD: Transanal excision for low rectal cancers is curative in early-stage disease with favorable histology. Am Surg 2000; 66:817-820

11 Miles W: A method of performing abdominoperineal excision for carcinoma of the rectum and of the terminal portion of the pelvic colon. Lancet 1908;ii: 1812 .

12 Goligher J: Treatment of Carcinoma of the Rectum. Surgery of the Anus, Rectum and Colon. London, Bailliere Tindall, 1984, pp 590779.

$\checkmark 13$ Goligher JC, Lee PW, MacFie J, Simkins KC, Lintott DJ: Experience with the Russian model 249 suture gun for anastomosis of the rectum. Surg Gynecol Obstet 1979;148:516-524.

14 Heald RJ, Husband EM, Ryall RD: The mesorectum in rectal cancer surgery - The clue to pelvic recurrence? Br J Surg 1982;69:613-616.

15 Camilleri-Brennan J, Steele RJ: Quality of life after treatment for rectal cancer. Br J Surg 1998;85:1036-1043

-16 Nicholls RJ, Mason AY, Morson BC, Dixon AK, Fry LK: The clinical staging of rectal cancer. Br J Surg 1982;69:404-409.

- 17 Gualdi GF, Casciani E, Guadalaxara A, et al Local staging of rectal cancer with transrectal ultrasound and endorectal magnetic resonance imaging. Dis Colon Rectum 2000;43:338-345.
18 Drew PJ, Farouk R, Turnbull LW, et al: Preoperative magnetic resonance staging of rectal cancer with an endorectal coil and dynamic gadolinium enhancement. Br J Surg 1999;86: 250-254

19 Matsuoka H, Nakamura A, Masaki T, et al: Comparison between endorectal coil and pelvic phased-array coli magnetic resonance imaging in patients with anorectal tumor. Am J Surg 2003; 185:328-332.

20 Garcia-Aguilar J, Mellgren A, Sirivongs P, et al: Local excision of rectal cancer without adjuvant therapy: A word of caution. Ann Surg 2000;231:345-351.

21 Brodsky JT, Richard GK, Cohen AM, Minsky BD: Variables correlated with the risk of lymph node metastasis in early rectal cancer. Cancer 1992;69:322-326.

22 Zenni GC, Abraham K, Harford FJ, et al: Characteristics of rectal carcinomas that predict the presence of lymph node metastases: Implications for patient selection for local therapy. J Surg Oncol 1998;67:99-103.

23 Kikuchi R, Takano M, Takagi K, et al: Management of early invasive rectal cancer. Risk of recurrence and clinical guidelines. Dis Colon Rectum 1995;38:1286-1295.

24 Willett CG, Compton CC, Shellito PC, Efird JT: Selection factors for local excision or abdominoperineal resection of early stage rectal cancer. Cancer 1994;73:2716-2720.

25 Balani A, Turoldo A, Braini A, et al: Local excision for rectal cancer. J Surg Oncol 2000;74: 158-162.

26 Buess G, Theiss R, Gunther M, Hutterer F, Pichlmaier H: Endoscopic surgery in the rectum. Endoscopy 1985;17:31-35.

27 Potenti F, Wexner SD: Local resection of rectal cancer. Hepatogastroenterology 2000;47:327334.

28 Coco C, Magistrelli P, Granone P, Roncolini $G$, Picciocchi A: Conservative surgery for early cancer of the distal rectum. Dis Colon Rectum 1992;35:131-136.

29 Frazee R, Patel R, Belew M, Roberts J, Hendricks J: Transanal excision of rectal carcinoma. Am Surg 1995;61:714-717.

30 Valentini V, Morganti AG, De Santis M, et al: Local excision and external beam radiotherapy in early rectal cancer. Int $\mathbf{J}$ Radiat Oncol Biol Phys 1996;35:759-764.

31 Le Voyer TE, Hoffman JP, Cooper H, et al: Local excision and chemoradiation for low rectal T1 and T2 cancers is an effective treatment. Am Surg 1999;65:625-630.

-32 Graham RA, Hackford AW, Wazer DE: Local excision of rectal carcinoma: a safe alternative for more advanced tumors? J Surg Oncol 1999; 70:235-238

33 Benson R, Wong CS, Cummings BJ, et al: Local excision and postoperative radiotherapy for distal rectal cancer. Int J Radiat Oncol Biol Phys 2001;50:1309-1316.
34 Chakravarti A, Compton CC, Shellito PC, et al: Long-term follow-up of patients with recta cancer managed by local excision with and without adjuvant irradiation. Ann Surg 1999; 230:49-54.

35 Varma MG, Rogers SJ, Schrock TR, Welton ML: Local excision of rectal carcinoma. Arch Surg 1999;134:863-867.

36 Wagman R, Minsky BD, Cohen AM, et al: Conservative management of rectal cancer with local excision and postoperative adjuvant therapy. Int J Radiat Oncol Biol Phys 1999;44: 841-846.

37 Taylor RH, Hay JH, Larsson SN: Transanal local excision of selected low rectal cancers. Am J Surg 1998;175:360-363.

38 Bleday R, Breen E, Jessup JM, et al: Prospective evaluation of local excision for small rectal cancers. Dis Colon Rectum 1997;40:388-392.

39 Fortunato L, Ahmad NR, Yeung RS, et al: Long-term follow-up of local excision and radiation therapy for invasive rectal cancer. Dis Colon Rectum 1995;38:1193-1199.

40 Steele GD Jr, Herndon JE, Bleday R, et al: Sphincter-sparing treatment for distal rectal adenocarcinoma. Ann Surg Oncol 1999;6:433441.

41 Ahmad NR, Nagle D: Long-term results of preoperative radiation therapy alone for stage T3 and T4 rectal cancer. Br J Surg 1997;84:14451448 .

42 Russell A, Harris J, Rosenberg P, et al: Anal sphincter conservation for patients with adenocarcinoma of the distal rectum: Long term results of radiation therapy oncology group protocol 89-02. Int J Radiat Oncol Biol Phys 2000;46:313-322.

43 Otmezguine Y, Grimard L, Calitchi E, et al: A new combined approach in the conservative management of rectal cancer. Int J Radiat Oncol Biol Phys 1989; 17:539-545.

44 Mohiuddin M, Marks G, Bannon J: High-dose preoperative radiation and full thickness local excision: A new option for selected T3 distal rectal cancers. Int J Radiat Oncol Biol Phys 1994:30:845-849.

45 Mohiuddin M, Regine WF, Marks GJ, Marks JW: High-dose preoperative radiation and the challenge of sphincter-preservation surgery for cancer of the distal $2 \mathrm{~cm}$ of the rectum. Int $\mathrm{J}$ Radiat Oncol Biol Phys 1998;40:569-574.

46 Kim CJ, Yeatman TJ, Coppola D, et al: Local excision of $\mathrm{T} 2$ and $\mathrm{T} 3$ rectal cancers after downstaging chemoradiation. Ann Surg 2001; 234:352-358.

47 Friel CM, Cromwell JW, Marra C, et al: Salvage radical surgery after failed local excision for early rectal cancer. Dis Colon Rectum 2002;45:875-879. 